\title{
Eigenfunctional representation of dyadic Green's functions in multilayered gyrotropic chiral media
}

\author{
Cheng-Wei Qiu ${ }^{1,2}$, Hai-Ying Yao ${ }^{1}$, Le-Wei Li ${ }^{1}$, Saïd Zouhdi ${ }^{2}$ \\ and Tat-Soon Yeo ${ }^{1}$ \\ ${ }^{1}$ Department of Electrical and Computer Engineering, National University of Singapore, \\ Kent Ridge, Singapore 117576, Singapore \\ ${ }^{2}$ Lab de Genie Electrique de Paris, CNRS, Ecole Superieure d'Electricite, \\ Plateau de Moulon 91192, Gif-sur-Yvette Cedex, France
}

Received 14 March 2007, in final form 16 April 2007

Published 8 May 2007

Online at stacks.iop.org/JPhysA/40/5751

\begin{abstract}
Studying electromagnetic waves in complex media has been an important research topic due to its useful applications and scientific significance of its physical performance. Dyadic Green's functions (DGFs), as a mathematical kernel or a dielectric medium response, have long been a valuable tool in solving both source-free and source-incorporated electromagnetic boundary value problems for electromagnetic scattering, radiation and propagation phenomena. A complete eigenfunctional expansion of the dyadic Green's functions for an unbounded and a planar, arbitrary multilayered gyrotropic chiral media is formulated in terms of the vector wavefunctions. After a general representation of Green's dyadics is obtained, the scattering coefficients of Green's dyadics are determined from the boundary conditions at each interface and are expressed in a greatly compact form of recurrence matrices. In the formulation of Green's dyadics and their scattering coefficients, three cases are considered, i.e. the current source is immersed in (1) the first, (2) the intermediate, and (3) the last regions, respectively. Although the dyadic Green's functions for an unbounded gyroelectric medium has been reported in the literature, we here present not only unbounded but also multilayered DGFs for the gyrotropic chiral media. The explicit representation of the DGFs after reduction to the gyroelectric or isotropic case agrees well with those existing corresponding results.
\end{abstract}

PACS number: 03.50.De

\section{Introduction}

Recently, negative refractive (NR) index materials have captured considerable attention in the research communities of physicists and engineers due to the exotic electromagnetic properties [1] and potential application in perfect lens [2]. After the negative refraction by the artificial materials was experimentally verified by Shelby [3], more studies on metamaterials 
have been carried out (e.g., tensor-parameter retrieval using quasi-static Lorentz theory [4], S-parameter retrieval using the plane wave incidence [5] and constitutive relation retrieval using transmission line method [6,7]). These materials may possess backward waves [8, 9], negative refraction [10, 11], subwavelength focusing [12] and amplification of evanescent waves [2]. However, the known realizations of metamaterials of a negative refraction index are based on the use of metal inclusions of different geometries such as complementary split rings [13], deformed split rings [14] and hexagon rings [15]. Most of the work reported are conducted in microwave regime, and thus to keep strong magnetic response in optical frequency becomes a challenge if optical applications are desired. In view of that, chiral media have been investigated, since the negative refraction can be achieved even if the material has no magnetic response properties. The backward waves have been studied in chiral nihility [16] and gyrotropic chiral media [17, 18]. It shows that gyrotropic chiral media provide more degrees of freedom to realize the negative refraction since the gyro-parameters would greatly reduce the refractive indices.

Different from the previous work on gyrotropic chiral media, our paper will focus on the formulation of dyadic Green's functions (DGFs) of gyrotropic chiral media, which relate directly the radiated electromagnetic fields and the source distribution. DGFs play an important role in solving both source-free and source-incorporated boundary value problems and in characterizing macroscopic performance of multilayered complex media [20, 25-27], and some special transformations dealing with the scattering and radiation phenomena are also proposed by Li et al in [28]. Nowadays, the dyadic Green's function technique has been an important method employed elsewhere for boundary value problems [29], such as in Method of Moments and Boundary Element Method. Since 1970s, DGFs in anisotropic media have been derived [30, 32-34] using (i) the Fourier transform technique, (ii) the method of angular spectrum expansion, and (iii) the transmission matrix method. However, complete formulation of the DGFs in various anisotropic media using eigenfunction expansion technique has not been achieved so far, though the DGFs for isotropic media have been studied quite well in the last three decades. The technique of Fourier transform looks simpler and is efficient for the Cartesian coordinates [20]; but it may not be applicable in all the nine fundamental coordinate systems. The technique of eigenfunctional expansion provides a systematic approach in electromagnetic theory for interpreting various electromagnetic representations [35]; more importantly, it is applicable in almost all the fundamental coordinates. Even in the planar structure considered in detail in this paper, the eigenfunctional expansion technique can provide an explicit form of the dyadic Green's functions in cylindrical Bessel functions, so that it becomes easy and convenient when the source distribution is independent of the azimuth directions or when far fields are computed.

There have been some results of DGFs for gyroelectric chiral media (only a subset of gyrotropic chiral media), however most of them are basically valid only for unbounded media $[36,37]$ while some of them are not correct, for instance, the results in [38] commented by Li et al [39]. Since gyrotropic chiral media exhibit potential applications in realization of negative-refractive media [17], the systematic characterization of such media is of our particular interest. In this paper, we not only consider the general gyrotropic chiral media but also take into account multilayered structures. This paper aims at (i) the direct development of the unbounded dyadic Green's functions in an unbounded gyrotropic chiral medium where the eigenfunction expansion technique is employed and mistakes in the existing work are pointed out; (ii) the formulations of the scattering dyadic Green's functions and their coefficients in planarly multilayered gyrotropic chiral media where each layer can be arbitrarily multiple stratified gyrotropic chiral medium and its results can be reduced to those of the isotropic or gyroelectric chiral media, and where the source is assumed to have an arbitrary 
three-dimensional distribution and either the transmitter or the receiver can be located in any region of the layered geometry; and (iii) the derivation of the irrotational part of the dyadic Green's functions which were not always provided in existing work. Due to the different geometries of the multilayered structures, the formulation of the dyadic Green's functions differs one from another.

Throughout the paper, a time dependence $\mathrm{e}^{-\mathrm{i} \omega t}$ is always suppressed.

\section{DGFs for unbounded gyrotropic chiral media}

A homogeneous gyrotropic chiral medium can be characterized by a set of constitutive relations

$$
\begin{aligned}
& \boldsymbol{D}=\overline{\boldsymbol{\epsilon}} \cdot \boldsymbol{E}+\mathrm{i} \xi_{c} B, \\
& \boldsymbol{H}=\mathrm{i} \xi_{c} \boldsymbol{E}+\overline{\boldsymbol{\mu}}^{-1} \cdot \boldsymbol{B},
\end{aligned}
$$

where

$$
\begin{aligned}
\overline{\boldsymbol{\epsilon}} & =\left[\begin{array}{lll}
\epsilon & -\mathrm{i} g & 0 \\
\mathrm{i} g & \epsilon & 0 \\
0 & 0 & \epsilon_{z}
\end{array}\right], \\
\overline{\boldsymbol{\mu}} & =\left[\begin{array}{lll}
\mu & -\mathrm{i} w & 0 \\
\mathrm{i} w & \mu & 0 \\
0 & 0 & \mu_{z}
\end{array}\right] .
\end{aligned}
$$

We note that gyrotropic chiral material can be reduced to gyroelectric (chiral objects embedded in a magnetic biased plasma) case or chiroferrite (chiral objects immersed in a magnetic biased ferrite) case [41]. Experimentally, there might be some problems of simultaneously controlling the gyro-parameters (i.e., $g$ and $w$ ). However, theoretical physics goes ahead often, relative to the experimental physics. Due to the generalization of the material discussed in this paper, it is valuable to investigate the DGFs for this material, especially in its multilayered structure.

\subsection{General formulation of unbounded DGFS}

Substituting (1) into the source-incorporated Maxwell's equations, we have

$$
\nabla \times\left[\overline{\boldsymbol{\mu}}^{-1} \cdot \nabla \times \boldsymbol{E}(\boldsymbol{r})\right]-2 \omega \xi_{c} \nabla \times \boldsymbol{E}(\boldsymbol{r})-\omega^{2} \overline{\boldsymbol{\epsilon}} \cdot \boldsymbol{E}(\boldsymbol{r})=\mathrm{i} \omega \boldsymbol{J}\left(\boldsymbol{r}^{\prime}\right) .
$$

Corresponding to the vector wave equation in (3), the following dyadic differential equation is obtained:

$\nabla \times\left[\overline{\boldsymbol{\mu}}^{-1} \cdot \nabla \times \overline{\boldsymbol{G}}_{e}\left(\boldsymbol{r}, \boldsymbol{r}^{\prime}\right)\right]-2 \omega \xi_{c} \nabla \times \overline{\boldsymbol{G}}_{e}\left(\boldsymbol{r}, \boldsymbol{r}^{\prime}\right)-\omega^{2} \overline{\boldsymbol{\epsilon}} \cdot \overline{\boldsymbol{G}}_{e}\left(\boldsymbol{r}, \boldsymbol{r}^{\prime}\right)=\overline{\boldsymbol{I}} \delta\left(\boldsymbol{r}-\boldsymbol{r}^{\prime}\right)$,

where $\overline{\boldsymbol{I}}$ and $\delta\left(\boldsymbol{r}-\boldsymbol{r}^{\prime}\right)$ denote the unit dyadic and Dirac delta function, respectively.

Substituting (3) and (4) into the vector-dyadic Green's theorem [19] and then applying the Sommerfeld radiation condition together with vector and tensor manipulations, we have the following solution to (3) due to the source $\boldsymbol{J}\left(\boldsymbol{r}^{\prime}\right)$ (which can be deduced from Weiglhofer's results [40] by assuming magnetic current density to be zero):

$$
\boldsymbol{E}(\boldsymbol{r})=\mathrm{i} \omega \int_{V^{\prime}} \overline{\boldsymbol{G}}_{e}\left(\boldsymbol{r}, \boldsymbol{r}^{\prime}\right) \cdot \boldsymbol{J}\left(\boldsymbol{r}^{\prime}\right) \mathrm{d} V^{\prime},
$$

where $V^{\prime}$ denotes the volume occupied by the source.

According to the Ohm-Rayleigh method, the source term in (5) can be expanded in terms of the solenoidal and irrotational cylindrical vector wavefunctions in cylindrical coordinates. 
Thus, we obtain

$$
\begin{aligned}
\overline{\boldsymbol{I}} \delta\left(\boldsymbol{r}-\boldsymbol{r}^{\prime}\right)= & \int_{0}^{\infty} \mathrm{d} \lambda \int_{-\infty}^{\infty} \mathrm{d} h \sum_{n=-\infty}^{\infty}\left[\boldsymbol{M}_{n}(h, \lambda) \boldsymbol{A}_{n}(h, \lambda)\right. \\
& \left.+\boldsymbol{N}_{n}(h, \lambda) \boldsymbol{B}_{n}(h, \lambda)+\boldsymbol{L}_{n}(h, \lambda) \boldsymbol{C}_{n}(h, \lambda)\right],
\end{aligned}
$$

where vector wavefunctions $M, N$ and $L$ in cylindrical coordinate system are defined as [19]

$$
\begin{aligned}
& M_{n}(h, \lambda)=\nabla \times\left[\Psi_{n}(h, \lambda) \widehat{z}\right], \\
& \boldsymbol{N}_{n}(h, \lambda)=\frac{1}{k_{\lambda}} \nabla \times M_{n}(h, \lambda), \\
& \boldsymbol{L}_{n}(h, \lambda)=\nabla\left[\Psi_{n}(h, \lambda)\right],
\end{aligned}
$$

where $k_{\lambda}=\sqrt{\lambda^{2}+h^{2}}$, and the generating function is given by $\Psi_{n}(h, \lambda)=J_{n}(\lambda \rho) \mathrm{e}^{\mathrm{i}(n \phi+h z)}$. The coefficients $\boldsymbol{A}_{n}(h, \lambda), \boldsymbol{B}_{n}(h, \lambda)$ and $\boldsymbol{C}_{n}(h, \lambda)$ in (6) are to be determined from the orthogonality relationships among the cylindrical vector wavefunctions. Therefore, scalar-dot multiply both sides of (6) with $\boldsymbol{M}_{-n^{\prime}}\left(-h^{\prime},-\lambda^{\prime}\right), \boldsymbol{N}_{-n^{\prime}}\left(-h^{\prime},-\lambda^{\prime}\right)$ and $\boldsymbol{L}_{-n^{\prime}}\left(-h^{\prime},-\lambda^{\prime}\right)$ each at a time and integrate them over the whole volume, and we obtain from the orthogonality that

$$
\begin{aligned}
& \boldsymbol{A}_{n}(h, \lambda)=\frac{1}{4 \pi^{2} \lambda} \boldsymbol{M}_{-n}^{\prime}(-h,-\lambda), \\
& \boldsymbol{B}_{n}(h, \lambda)=\frac{1}{4 \pi^{2} \lambda} \boldsymbol{N}_{-n}^{\prime}(-h,-\lambda), \\
& \boldsymbol{C}_{n}(h, \lambda)=\frac{\lambda}{4 \pi^{2}\left(\lambda^{2}+h^{2}\right)} \boldsymbol{L}_{-n}^{\prime}(-h,-\lambda) .
\end{aligned}
$$

As is known to all, the dyadic Green's function can thus be expanded as follows:

$$
\begin{aligned}
\overline{\boldsymbol{G}}_{0}\left(\boldsymbol{r}, \boldsymbol{r}^{\prime}\right)= & \int_{0}^{\infty} \mathrm{d} \lambda \int_{-\infty}^{\infty} \mathrm{d} h \sum_{n=-\infty}^{\infty}\left[\boldsymbol{M}_{n}(h, \lambda) \boldsymbol{a}_{n}(h, \lambda)\right. \\
& \left.+\boldsymbol{N}_{n}(h, \lambda) \boldsymbol{b}_{n}(h, \lambda)+\boldsymbol{L}_{n}(h, \lambda) \boldsymbol{c}_{n}(h, \lambda)\right],
\end{aligned}
$$

where the vector expansion coefficients $\boldsymbol{a}_{n}(h, \lambda), \boldsymbol{b}_{n}(h, \lambda)$ and $\boldsymbol{c}_{n}(h, \lambda)$ are unknown vector coefficients to be determined from the orthogonality and permittivity and permeability tensors' properties.

To obtain these unknown vectors, we substitute (9) and (6) into (5), noting the instinct properties of the vector wavefunctions

$$
\begin{aligned}
& \nabla \times N_{n}(h, \lambda)=k_{\lambda} M_{n}(h, \lambda), \\
& \nabla \times M_{n}(h, \lambda)=k_{\lambda} N_{n}(h, \lambda), \\
& \nabla \times L_{n}(h, \lambda)=0 .
\end{aligned}
$$

We can thus obtain

$$
\begin{aligned}
& \int_{0}^{\infty} \mathrm{d} \lambda \int_{-\infty}^{\infty} \mathrm{d} h \sum_{n=-\infty}^{\infty}\left[\boldsymbol{M}_{n}(h, \lambda) \boldsymbol{A}_{n}(h, \lambda)+\boldsymbol{N}_{n}(h, \lambda) \boldsymbol{B}_{n}(h, \lambda)+\boldsymbol{L}_{n}(h, \lambda) \boldsymbol{C}_{n}(h, \lambda)\right] \\
&=\int_{0}^{\infty} \mathrm{d} \lambda \int_{-\infty}^{\infty} \mathrm{d} h \sum_{n=-\infty}^{\infty}\left\{\nabla \times\left[\overline{\boldsymbol{\mu}}^{-1} \cdot \boldsymbol{k}_{\lambda}\left(\boldsymbol{N}_{n}(h, \lambda) \boldsymbol{a}_{n}(h, \lambda)+\boldsymbol{M}_{n}(h, \lambda) \boldsymbol{b}_{n}(h, \lambda)\right)\right]\right. \\
&-2 k_{\lambda} \omega \xi_{c}\left[\boldsymbol{N}_{n}(h, \lambda) \boldsymbol{a}_{n}(h, \lambda)+\boldsymbol{M}_{n}(h, \lambda) \boldsymbol{b}_{n}(h, \lambda)\right] \\
&\left.-\omega^{2} \overline{\boldsymbol{\epsilon}} \cdot\left[\boldsymbol{M}_{n}(h, \lambda) \boldsymbol{a}_{n}(h, \lambda)+\boldsymbol{N}_{n}(h, \lambda) \boldsymbol{b}_{n}(h, \lambda)+\boldsymbol{L}_{n}(h, \lambda) \boldsymbol{c}_{n}(h, \lambda)\right]\right\}
\end{aligned}
$$


For the compactness of the coming manipulation, we define

$$
\overline{\boldsymbol{\alpha}}=\overline{\boldsymbol{\mu}}^{-1}=\left[\begin{array}{ccc}
\alpha_{t} & -\alpha_{a} & 0 \\
\alpha_{a} & \alpha_{t} & 0 \\
0 & 0 & \alpha_{z}
\end{array}\right]
$$

where

$$
\alpha_{t}=\frac{\mu}{\mu^{2}-w^{2}}, \quad \alpha_{a}=\frac{-\mathrm{i} w}{\mu^{2}-w^{2}}, \quad \alpha_{z}=\frac{1}{\mu_{z}} .
$$

By taking respectively the anterior scalar product of (11) with the vector wave equations and performing the integration over the entire volume, we can formulate the equations satisfied by the unknown vectors and the known scalar and vector parameters in a matrix form as given by

$$
\left[\begin{array}{l}
\Phi_{1} \\
\Phi_{2} \\
\Phi_{3}
\end{array}\right][X]=[\Theta]
$$

where $\left[\Phi_{i}\right]$ denotes $i$ th row in a $3 \times 3$ matrix

$$
\begin{aligned}
& \Phi_{1}=\left[h^{2} \alpha_{t}+\lambda^{2} \alpha_{z}-\omega^{2} \epsilon,-\left(\frac{\mathrm{i} \omega^{2} h g}{k_{\lambda}}+\mathrm{i} k_{\lambda} h \alpha_{a}+2 \omega \xi_{c} k_{\lambda}\right), \mathrm{i} \omega^{2} g\right], \\
& \Phi_{2}=\left[-\left(\frac{\mathrm{i} \omega^{2} h g}{k_{\lambda}}+\mathrm{i} k_{\lambda} h \alpha_{a}+2 \omega \xi_{c} k_{\lambda}\right), k_{\lambda}^{2} \alpha_{t}-\omega^{2} \frac{h^{2} \epsilon+\lambda^{2} \epsilon_{z}}{k_{\lambda}^{2}},-\omega^{2} \frac{\mathrm{i} h}{k_{\lambda}}\left(\epsilon_{z}-\epsilon\right)\right], \\
& \Phi_{3}=\left[-\mathrm{i} \omega^{2} \frac{\lambda^{2}}{k_{\lambda}^{2}} g, \omega^{2} \frac{\mathrm{i} h \lambda^{2}}{k_{\lambda}^{3}}\left(\epsilon_{z}-\epsilon\right),-\omega^{2} \frac{h^{2} \epsilon_{z}+\lambda^{2} \epsilon}{k_{\lambda}^{2}}\right] .
\end{aligned}
$$

$[X]$ and $[\Theta]$ are known column vectors, $[X]=\left[\boldsymbol{a}_{n}(h, \lambda) \boldsymbol{b}_{n}(h, \lambda) \boldsymbol{c}_{n}(h, \lambda)\right]^{T}$ and $[\Theta]=$ $\left[\boldsymbol{A}_{n}(h, \lambda) \boldsymbol{B}_{n}(h, \lambda) \boldsymbol{C}_{n}(h, \lambda)\right]^{T}$. Solving (14), we have the solutions for $\boldsymbol{a}_{n}(h, \lambda), \boldsymbol{b}_{n}(h, \lambda)$ and $c_{n}(h, \lambda)$ as

$$
\begin{aligned}
& \boldsymbol{a}_{n}(h, \lambda)=\frac{1}{\Gamma}\left[\alpha_{1} \boldsymbol{A}_{n}(h, \lambda)+\beta_{1} \boldsymbol{B}_{n}(h, \lambda)+\gamma_{1} \boldsymbol{C}_{n}(h, \lambda)\right], \\
& \boldsymbol{b}_{n}(h, \lambda)=\frac{1}{\Gamma}\left[\alpha_{2} \boldsymbol{A}_{n}(h, \lambda)+\beta_{2} \boldsymbol{B}_{n}(h, \lambda)+\gamma_{2} \boldsymbol{C}_{n}(h, \lambda)\right], \\
& \boldsymbol{c}_{n}(h, \lambda)=\frac{1}{\Gamma}\left[\alpha_{3} \boldsymbol{A}_{n}(h, \lambda)+\beta_{3} \boldsymbol{B}_{n}(h, \lambda)+\gamma_{3} \boldsymbol{C}_{n}(h, \lambda)\right],
\end{aligned}
$$

where

$$
\Gamma=\epsilon_{z} \alpha_{t}\left(k_{\lambda}^{2}-k_{1}^{2}\right)\left(k_{\lambda}^{2}-k_{2}^{2}\right) / \alpha_{z}
$$

and

$$
k_{1,2}^{2}=\frac{1}{2 \epsilon_{z} \alpha_{t} / \alpha_{z}}\left[-p_{\lambda} \pm \sqrt{p_{\lambda}^{2}+4 \epsilon_{z} \alpha_{t} / \alpha_{z} q_{\lambda}}\right]
$$

with $p_{\lambda}$ and $q_{\lambda}$ given respectively below:

$$
\begin{gathered}
p_{\lambda}=\left[\left(\alpha_{t} / \alpha_{z}\right)^{2}+\left(\alpha_{a} / \alpha_{z}\right)^{2}\right] h^{2} \epsilon-4 \mathrm{i} h \epsilon \xi_{c} \omega \alpha_{a} / \alpha_{z}^{2} \\
-\left[4 \epsilon\left(\xi_{c} / \alpha_{z}\right)^{2}+\epsilon \epsilon_{z} / \alpha_{z}\right] \omega^{2}+\left(g^{2}-\epsilon^{2}\right) \omega^{2} \alpha_{t} / \alpha_{z}^{2}-h^{2} \epsilon_{z} \alpha_{t} / \alpha_{z} \\
q_{\lambda}=-\left[\left(\alpha_{t} / \alpha_{z}\right)^{2}+\left(\alpha_{a} / \alpha_{z}\right)^{2}\right] h^{4} \epsilon_{z}+4 \mathrm{i} h^{2}\left(2 h \xi_{c}+g \omega\right) \epsilon_{z} \omega \alpha_{a} / \alpha_{z}^{2} \\
+\frac{\epsilon_{z}}{\alpha_{z}^{2}}\left[4 h^{2} \xi_{c}^{2}+4 g h \xi_{c} \omega+\left(g^{2}-\epsilon^{2}\right) \omega^{2}+2 \alpha_{t} h^{2} \epsilon \epsilon_{z}\right] \omega^{2} .
\end{gathered}
$$


It should be noted that the coupling coefficients $\beta_{1}, \gamma_{1}, \alpha_{2}, \gamma_{2}, \alpha_{3}$ and $\beta_{3}$ were assumed to be zero in [38]. Here it is proved that those coupling coefficients must be considered in the formulation since they are not always zero, and the coupling coefficients $\alpha_{1,2,3}, \beta_{1,2,3}$ and $\gamma_{1,2,3}$ are given in detail below

$$
\begin{aligned}
\alpha_{1}= & \frac{\alpha_{t}}{\alpha_{z}^{2}}\left(h^{2} \epsilon_{z}+\lambda^{2} \epsilon\right)-\frac{1}{\alpha_{z}^{2}} \omega^{2} \epsilon \epsilon_{z}, \\
\alpha_{2}= & \beta_{1}=\frac{1}{k_{\lambda} \alpha_{z}^{2}}\left[\mathrm{i} h \alpha_{a}\left(h^{2} \epsilon_{z}+\lambda^{2} \epsilon\right)+2 \xi_{c}\left(h^{2} \epsilon_{z}+\epsilon \lambda^{2}\right) \omega+h g \epsilon_{z} \omega^{2}\right], \\
\gamma_{1}= & -\frac{k_{\lambda}^{2}}{\lambda^{2}} \alpha_{3}=\frac{\mathrm{i}}{\alpha_{z}^{2}}\left[g k_{\lambda}^{2} \alpha_{t}+\mathrm{i} h^{2} \alpha_{a}\left(\epsilon-\epsilon_{z}\right)+2 h \xi_{c}\left(\epsilon-\epsilon_{z}\right) \omega-g \epsilon_{z} \omega^{2}\right], \\
\gamma_{2}= & -\frac{k_{\lambda}^{2}}{\lambda^{2}} \beta_{3} \\
= & \frac{\mathrm{i}}{k_{\lambda}}\left[h\left(h^{2} \frac{\alpha_{t}}{\alpha_{z}}+\lambda^{2}\right)\left(\epsilon-\epsilon_{z}\right) / \alpha_{z}+\mathrm{i} g h k_{\lambda}^{2} \alpha_{a} / \alpha_{z}^{2}\right. \\
& \left.+2 g \xi_{c} k_{\lambda}^{2} \omega / \alpha_{z}^{2}-h\left(\epsilon^{2}-\epsilon \epsilon_{z}-g^{2}\right) \omega^{2} / \alpha_{z}^{2}\right], \\
\beta_{2}= & \frac{1}{k_{\lambda}^{2}}\left[\left(h^{2} \frac{\alpha_{t}}{\alpha_{z}}-\lambda^{2}\right)\left(h^{2} \epsilon_{z}+\lambda^{2} \epsilon\right) / \alpha_{z}-\left(h^{2} \epsilon \epsilon_{z}+\lambda^{2}\left(\epsilon^{2}-g^{2}\right)\right) \omega^{2} / \alpha_{z}^{2}\right] \\
\gamma_{3}= & \frac{1}{\omega^{2}}\left\{-k_{\lambda}^{2}\left(h^{2} \frac{\alpha_{t}^{2}+\alpha_{a}^{2}}{\alpha_{z}^{2}}+\lambda^{2} \frac{\alpha_{t}}{\alpha_{z}}\right)+4 \mathrm{i} h k_{\lambda}^{2} \alpha_{a} \xi_{c} \omega / \alpha_{z}^{2}\right. \\
& +\left[k_{\lambda}^{2} \epsilon \alpha_{t}+\frac{h^{2} \alpha_{t}+\lambda^{2} \alpha_{z}}{k_{\lambda}^{2}}\left(h^{2} \epsilon+\lambda^{2} \epsilon_{z}\right)+2 \mathrm{i} h^{2} g \alpha_{a}+4 k_{\lambda}^{2} \xi_{c}^{2}\right] \omega^{2} / \alpha_{z}^{2} \\
& \left.+4 g h \xi_{c} \omega^{3} / \alpha_{z}^{2}+\frac{1}{k_{\lambda}^{2}}\left[h^{2}\left(g^{2}-\epsilon^{2}\right)-\lambda^{2} \epsilon \epsilon_{z}\right] \omega^{4} / \alpha_{z}^{2}\right\} .
\end{aligned}
$$

Hence, the unbounded DGFs in (9) can be rewritten as

$$
\begin{aligned}
\overline{\boldsymbol{G}}_{0}\left(\boldsymbol{r}, \boldsymbol{r}^{\prime}\right)= & \int_{-\infty}^{\infty} \mathrm{d} h \int_{0}^{\infty} \mathrm{d} \lambda \sum_{n=-\infty}^{\infty} \frac{1}{4 \pi^{2} \lambda \Gamma} \\
& \times\left\{\boldsymbol{M}_{n}(h, \lambda)\left[\alpha_{1} \boldsymbol{M}_{-n}^{\prime}(-h,-\lambda)+\beta_{1} \boldsymbol{N}_{-n}^{\prime}(-h,-\lambda)+\frac{\lambda^{2}}{k_{\lambda}^{2}} \gamma_{1} \boldsymbol{L}_{-n}^{\prime}(-h,-\lambda)\right]\right. \\
& +\boldsymbol{N}_{n}(h, \lambda)\left[\beta_{1} \boldsymbol{M}_{-n}(-h,-\lambda)+\beta_{2} \boldsymbol{N}_{-n}(-h,-\lambda)+\frac{\lambda^{2}}{k_{\lambda}^{2}} \gamma_{2} \boldsymbol{L}_{-n}(-h,-\lambda)\right] \\
& \left.+\frac{\lambda^{2}}{k_{\lambda}^{2}} \boldsymbol{L}_{n}(h, \lambda)\left[-\gamma_{1} \boldsymbol{M}_{-n}(-h,-\lambda)-\gamma_{2} \boldsymbol{N}_{-n}(-h,-\lambda)+\gamma_{3} \boldsymbol{L}_{-n}(-h,-\lambda)\right]\right\} .
\end{aligned}
$$

We can further express (9) in a more compact form

$$
\begin{aligned}
\overline{\boldsymbol{G}}_{0}\left(\boldsymbol{r}, \boldsymbol{r}^{\prime}\right)= & \int_{-\infty}^{\infty} \mathrm{d} h \int_{0}^{\infty} \mathrm{d} \lambda \sum_{n=-\infty}^{\infty} \frac{1}{4 \pi^{2} \lambda \Gamma}\left\{\tau_{1} \boldsymbol{M}_{n}(h, \lambda) \boldsymbol{M}_{-n}^{\prime}(-h,-\lambda)\right. \\
& +\tau_{2}\left[\boldsymbol{M}_{n}(h, \lambda) \boldsymbol{N}_{-n t}^{\prime}(-h,-\lambda)+\boldsymbol{N}_{n t}(h, \lambda) \boldsymbol{M}_{-n}^{\prime}(-h,-\lambda)\right] \\
& +\tau_{3}\left[\boldsymbol{M}_{n}(h, \lambda) \boldsymbol{N}_{-n z}^{\prime}(-h,-\lambda)+\boldsymbol{N}_{n z}(h, \lambda) \boldsymbol{M}_{-n}^{\prime}(-h,-\lambda)\right] \\
& +\tau_{4}\left[\boldsymbol{N}_{n t}(h, \lambda) \boldsymbol{N}_{-n z}^{\prime}(-h,-\lambda)+\boldsymbol{N}_{n z}(h, \lambda) \boldsymbol{N}_{-n t}^{\prime}(-h,-\lambda)\right] \\
& \left.+\tau_{5} \boldsymbol{N}_{n t}(h, \lambda) \boldsymbol{N}_{-n t}^{\prime}(-h,-\lambda)+\tau_{6} \boldsymbol{N}_{n z}(h, \lambda) \boldsymbol{N}_{-n z}^{\prime}(-h,-\lambda)\right\},
\end{aligned}
$$


where

$$
\begin{aligned}
\tau_{1} & =\alpha_{1} \\
\tau_{2} & =\beta_{1}+\frac{\mathrm{i} \lambda^{2}}{k_{\lambda} h} \gamma_{1} \\
\tau_{3} & =\beta_{1}-\frac{\mathrm{i} h}{k_{\lambda}} \gamma_{1} \\
\tau_{4} & =\beta_{2}-\frac{\mathrm{i} h}{k_{\lambda}} \gamma_{2}-\frac{\mathrm{i} k_{\lambda}}{h} \beta_{3}-\gamma_{3} \\
\tau_{5} & =\beta_{2}+\frac{\mathrm{i} \lambda^{2}}{k_{\lambda} h} \gamma_{2}-\frac{\mathrm{i} k_{\lambda}}{h} \beta_{3}+\frac{\lambda^{2}}{h^{2}} \gamma_{3} \\
\tau_{6} & =\beta_{2}-\frac{\mathrm{i} h}{k_{\lambda}} \gamma_{2}+\frac{\mathrm{i} h k_{\lambda}}{\lambda^{2}} \beta_{3}+\frac{h^{2}}{\lambda^{2}} \gamma_{3} .
\end{aligned}
$$

It is worth noting that the following identities have been used in the derivation from (9) to (28)

$$
\begin{aligned}
& \boldsymbol{L}_{n}(h, \lambda)=\boldsymbol{L}_{n t}(h, \lambda)+\boldsymbol{L}_{n z}(h, \lambda), \\
& \boldsymbol{L}_{-n}^{\prime}(-h,-\lambda)=\boldsymbol{L}_{-n t}^{\prime}(-h,-\lambda)+\boldsymbol{L}_{-n z}^{\prime}(-h,-\lambda), \\
& \boldsymbol{N}_{n}(h, \lambda)=\boldsymbol{N}_{n t}(h, \lambda)+\boldsymbol{N}_{n z}(h, \lambda), \\
& \boldsymbol{N}_{-n}^{\prime}(-h,-\lambda)=\boldsymbol{N}_{-n t}^{\prime}(-h,-\lambda)+\boldsymbol{N}_{-n z}^{\prime}(-h,-\lambda), \\
& \boldsymbol{L}_{n t}(h, \lambda)=\frac{-\mathrm{i} k_{\lambda}}{h} \boldsymbol{N}_{n t}(h, \lambda), \\
& \boldsymbol{L}_{-n t}^{\prime}(-h,-\lambda)=\frac{\mathrm{i} k_{\lambda}}{h} \boldsymbol{N}_{-n t}^{\prime}(-h,-\lambda), \\
& \boldsymbol{L}_{n z}(h, \lambda)=\frac{\mathrm{i} h k_{\lambda}}{\lambda^{2}} \boldsymbol{N}_{n z}(h, \lambda), \\
& \boldsymbol{L}_{-n z}^{\prime}(-h,-\lambda)=\frac{-\mathrm{i} h k_{\lambda}}{\lambda^{2}} \boldsymbol{N}_{-n z}^{\prime}(-h,-\lambda),
\end{aligned}
$$

where the subscripts $t$ and $z$ denote, respectively, the transverse and the longitude components of the two functions $\boldsymbol{L}_{n}(h, \lambda)$ and $\boldsymbol{N}_{n}(h, \lambda)$ and similarly for the primed functions.

\subsection{Analytical evaluation of the $h$ integral}

In order to apply the residue theorem to (28), the part in (28) which does not satisfy the Jordan lemma is required to be extracted first. By applying the idea of Tai [19] to obtain an exact expression of the irrotational dyadic Green's function, we obtain from (6)

$\widehat{\boldsymbol{z}} \widehat{\boldsymbol{z}} \delta\left(\boldsymbol{r}-\boldsymbol{r}^{\prime}\right)=\int_{0}^{\infty} \mathrm{d} \lambda \int_{-\infty}^{\infty} \mathrm{d} h \sum_{n=-\infty}^{\infty} \frac{1}{4 \pi^{2} \lambda} \frac{k_{\lambda}^{2}}{\lambda^{2}} \boldsymbol{N}_{n z}(h, \lambda) \boldsymbol{N}_{-n z}^{\prime}(-h,-\lambda)$.

Thus the singular term of the unbounded DGF is contained in the $\boldsymbol{N}_{n z}(h, \lambda) N_{-n z}^{\prime}(-h,-\lambda)$ dyadic.

Certainly, the expression of the DGF, as shown above, is a form in a pre-integration domain. To actually make use of it for practical problems, we need to integrate the DGF in the pre-integration domain using the contour integration. In this paper, planarly multilayered structures are considered. As a result, the wave numbers $h_{i}$ can be found by rewriting $\Gamma$ in (18) in $h$-domain and solving the fourth-order polynomial equation. It can be obtained using

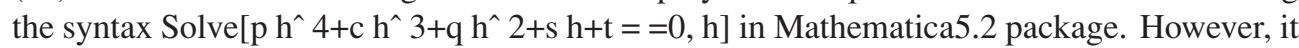


is too tedious to be shown here. One important point is that $\Gamma=0$ yields four sets of solutions corresponding to four different waves of wave numbers $h_{i}(i=1,2,3$ and 4$)$.

After lengthy but careful algebraic manipulation, we rewrite (28)

$$
\begin{aligned}
\overline{\boldsymbol{G}}_{0}\left(\boldsymbol{r}, \boldsymbol{r}^{\prime}\right)=- & \frac{\alpha_{z}}{\omega^{2} \epsilon_{z} \alpha_{t}} \widehat{\boldsymbol{z}} \widehat{\boldsymbol{z}} \delta\left(\boldsymbol{r}-\boldsymbol{r}^{\prime}\right)+\int_{-\infty}^{\infty} \mathrm{d} h \int_{0}^{\infty} \mathrm{d} \lambda \sum_{n=-\infty}^{\infty} \frac{1}{4 \pi^{2} \lambda \Gamma}\left\{\tau_{1} \boldsymbol{M}_{n}(h, \lambda) \boldsymbol{M}_{-n}^{\prime}(-h,-\lambda)\right. \\
& +\tau_{2}\left[\boldsymbol{M}_{n}(h, \lambda) \boldsymbol{N}_{-n t}^{\prime}(-h,-\lambda)+\boldsymbol{N}_{n t}(h, \lambda) \boldsymbol{M}_{-n}^{\prime}(-h,-\lambda)\right] \\
& +\tau_{3}\left[\boldsymbol{M}_{n}(h, \lambda) \boldsymbol{N}_{-n z}^{\prime}(-h,-\lambda)+\boldsymbol{N}_{n z}(h, \lambda) \boldsymbol{M}_{-n}^{\prime}(-h,-\lambda)\right] \\
& +\tau_{4}\left[\boldsymbol{N}_{n t}(h, \lambda) \boldsymbol{N}_{-n z}^{\prime}(-h,-\lambda)+\boldsymbol{N}_{n z}(h, \lambda) \boldsymbol{N}_{-n t}^{\prime}(-h,-\lambda)\right] \\
& \left.+\tau_{5} \boldsymbol{N}_{n t}(h, \lambda) \boldsymbol{N}_{-n t}^{\prime}(-h,-\lambda)+\tau_{7} \boldsymbol{N}_{n z}(h, \lambda) \boldsymbol{N}_{-n z}^{\prime}(-h,-\lambda)\right\}
\end{aligned}
$$

where

$\tau_{7}=\beta_{2}+\frac{1}{\omega^{2}}\left(1+h^{2} / \lambda^{2}\right)\left(k_{\lambda}^{2}-k_{1}^{2}\right)\left(k_{\lambda}^{2}-k_{2}^{2}\right)+\frac{h}{\lambda^{2}}\left(\mathrm{i} k_{\lambda} \beta_{3}+h \gamma_{3}\right)-\frac{\mathrm{i} h}{k_{\lambda}} \gamma_{2}$.

For $z>z^{\prime}$, the DGF is given by

$$
\begin{aligned}
\overline{\boldsymbol{G}}_{0}\left(\boldsymbol{r}, \boldsymbol{r}^{\prime}\right)=- & \frac{\alpha_{z}}{\omega^{2} \epsilon_{z} \alpha_{t}} \widehat{\boldsymbol{z}} \widehat{\boldsymbol{z}} \delta\left(\boldsymbol{r}-\boldsymbol{r}^{\prime}\right)+\frac{\mathrm{i}}{2 \pi} \int_{0}^{\infty} \mathrm{d} \lambda \sum_{n=-\infty}^{\infty} \frac{\alpha_{z}}{\epsilon_{z} \alpha_{t} \lambda\left(h_{1}-h_{2}\right)} \\
& \times \sum_{j=1}^{2} \frac{(-1)^{j+1}}{\left(h_{j}-h_{3}\right)\left(h_{j}-h_{4}\right)}\left\{\boldsymbol{M}_{n, \lambda}\left(h_{j}\right) \boldsymbol{P}_{-n,-\lambda}^{\prime}\left(-h_{j}\right)+\boldsymbol{Q}_{n, \lambda}\left(h_{j}\right) \boldsymbol{M}_{-n,-\lambda}^{\prime}\left(-h_{j}\right)\right. \\
& \left.+\boldsymbol{U}_{n, \lambda}\left(h_{j}\right) \boldsymbol{N}_{-n t,-\lambda}^{\prime}\left(-h_{j}\right)+\boldsymbol{V}_{n, \lambda}\left(h_{j}\right) \boldsymbol{N}_{-n z,-\lambda}^{\prime}\left(-h_{j}\right)\right\} .
\end{aligned}
$$

For $z<z^{\prime}$, the only replacement to be made is

$$
\frac{1}{\lambda\left(h_{1}-h_{2}\right)} \sum_{j=1}^{2} \frac{(-1)^{j+1}}{\left(h_{j}-h_{3}\right)\left(h_{j}-h_{4}\right)} \Longrightarrow \frac{1}{\lambda\left(h_{3}-h_{4}\right)} \sum_{j=3}^{4} \frac{(-1)^{j+1}}{\left(h_{1}-h_{j}\right)\left(h_{2}-h_{j}\right)} \text {. }
$$

The vector wavefunctions $\boldsymbol{P}_{n, \lambda}\left(h_{j}\right), \boldsymbol{Q}_{n, \lambda}\left(h_{j}\right), \boldsymbol{U}_{n, \lambda}\left(h_{j}\right)$ and $\boldsymbol{V}_{n, \lambda}\left(h_{j}\right)$ in (34), are given respectively by

$$
\begin{aligned}
& \boldsymbol{P}_{n, \lambda}\left(h_{j}\right)=\tau_{1} \boldsymbol{M}_{n, \lambda}\left(h_{j}\right)+\tau_{2} \boldsymbol{N}_{n t, \lambda}\left(h_{j}\right)+\tau_{3} \boldsymbol{N}_{n z, \lambda}\left(h_{j}\right), \\
& \boldsymbol{Q}_{n, \lambda}\left(h_{j}\right)=\tau_{2} \boldsymbol{N}_{n t, \lambda}\left(h_{j}\right)+\tau_{3} \boldsymbol{N}_{n z, \lambda}\left(h_{j}\right), \\
& \boldsymbol{U}_{n, \lambda}\left(h_{j}\right)=\tau_{5} \boldsymbol{N}_{n t, \lambda}\left(h_{j}\right)+\tau_{4} \boldsymbol{N}_{n z, \lambda}\left(h_{j}\right), \\
& \boldsymbol{V}_{n, \lambda}\left(h_{j}\right)=\tau_{4} \boldsymbol{N}_{n t, \lambda}\left(h_{j}\right)+\tau_{7} \boldsymbol{N}_{n z, \lambda}\left(h_{j}\right) .
\end{aligned}
$$

The first term of (34) is due to the contribution from the non-solenoidal vector wavefunctions. For a long time, this term of the DGF expressions was never been obtained in the publications $[43,44]$ where the irrotational part of the DGF was all missing. The second integration term can be evaluated by making use of the residue theorem in $h$-plane. This term contributes from the solenoidal vector wavefunctions. After some mathematical manipulations for simplicity, we arrived at the final representation of the DGFs for an unbounded gyrotropic chiral medium which is suitable for further analysis of a planar, multilayered structure.

Now, a complete representation of the DGFs for an unbounded gyrotropic chiral medium has been obtained. It can be seen that a singularity term has been extracted and this general DGFs are reducible to those of anisotropic, gyroelectric, chiroferrite and isotropic media. 


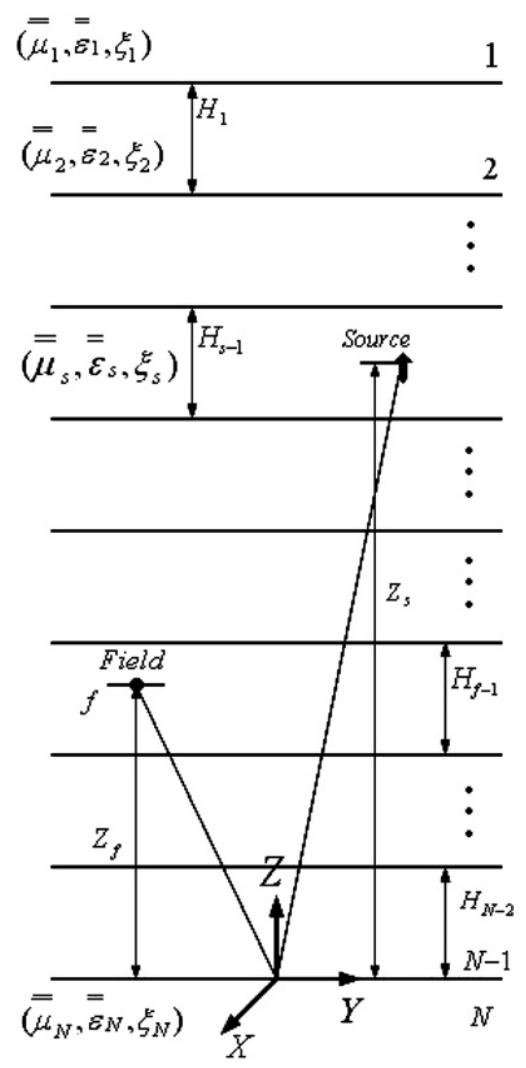

Figure 1. Geometry of a planarly multilayered Gyrotropic chiral medium.

\section{General formulation for stratified, multilayered gyrotropic chiral media}

In this section, we extend our theoretical analysis to the multilayered planar structure where both the number of multiple layers and the location of either the source or the field are arbitrary, and each layer can be a gyroelectric chiral (permeability is a scalar), a chiroferrite (permittivity is a scalar), a chiral (permittivity and permeability are both scalar), or just simply an isotropic medium $\left(\xi_{c}=0\right.$, and permittivity and permeability are both scalar). In view of practical fabrication of such multilayers, the bisoliton theory can be applied in the process [45]. The permittivity and permeability tensors are given below:

$$
\overline{\boldsymbol{\epsilon}}^{f}=\left[\begin{array}{lll}
\epsilon^{f} & -\mathrm{i} g^{f} & 0 \\
\mathrm{i} g^{f} & \epsilon^{f} & 0 \\
0 & 0 & \epsilon_{z}^{f}
\end{array}\right], \quad \overline{\boldsymbol{\mu}}^{f}=\left[\begin{array}{lll}
\mu^{f} & -\mathrm{i} w^{f} & 0 \\
\mathrm{i} w^{f} & \mu^{f} & 0 \\
0 & 0 & \mu_{z}^{f}
\end{array}\right],
$$

and correspondingly the matrix $\overline{\boldsymbol{\alpha}}^{f}=\left[\overline{\boldsymbol{\mu}}^{f}\right]^{-1}$ and the chiral parameter $\xi_{c f}$ in the $f$ th layer. Thus, the relation governing the wave number and eigenvalues can now be rewritten as

$$
k_{\lambda}^{f}=\sqrt{\lambda^{2}+\left(h^{f}\right)^{2}} .
$$

The multilayered structure is shown by figure 1 . 
Based on the principle of scattering superposition, we have

$$
\overline{\boldsymbol{G}}_{e}^{(f s)}\left(\boldsymbol{r}, \boldsymbol{r}^{\prime}\right)=\overline{\boldsymbol{G}}_{0}\left(\boldsymbol{r}, \boldsymbol{r}^{\prime}\right) \delta_{f}^{s}+\overline{\boldsymbol{G}}_{s}^{(f s)}\left(\boldsymbol{r}, \boldsymbol{r}^{\prime}\right),
$$

where $\overline{\boldsymbol{G}}_{e}$ and $\overline{\boldsymbol{G}}_{0}$ denote the total and unbounded electric DGFs, respectively and superscripts/subscripts $f$ and $s$ denote the field point located at the $f$ th layer and source located at the sth layer, respectively.

The representation of the scattered DGFs is given by

$$
\overline{\boldsymbol{G}}_{s}^{(f s)}\left(\boldsymbol{r}, \boldsymbol{r}^{\prime}\right)=\sum_{j=1}^{4} \overline{\boldsymbol{G}}_{j} .
$$

We combine two terms as follows:

$$
\begin{aligned}
\overline{\boldsymbol{G}}_{1}+ & \overline{\boldsymbol{G}}_{2}=\frac{\mathrm{i}}{2 \pi} \int_{0}^{\infty} \mathrm{d} \lambda \sum_{n=0}^{\infty} \frac{\left(2-\delta_{n 0}\right)}{p_{s} \lambda\left(h_{1 s}-h_{2 s}\right)} \times \sum_{j=1}^{2} \frac{(-1)^{j+1}}{\left(h_{j s}-h_{3 s}\right)\left(h_{j s}-h_{4 s}\right)} \\
& \times\left\{\left(1-\delta_{f}^{N}\right) \boldsymbol{M}_{n, \lambda}\left(h_{j}^{f}\right)\left[\left(1-\delta_{s}^{1}\right) A_{M j}^{f s} \boldsymbol{P}_{-n,-\lambda}^{\prime}\left(-h_{j}^{s}\right)+\left(1-\delta_{s}^{N}\right) B_{M j}^{f s} \boldsymbol{P}_{-n,-\lambda}^{\prime}\left(-h_{j+2}^{s}\right)\right]\right. \\
& +\left(1-\delta_{f}^{N}\right) \boldsymbol{Q}_{n, \lambda}\left(h_{j}^{f}\right)\left[\left(1-\delta_{s}^{1}\right) A_{Q j}^{f s} \boldsymbol{M}^{\prime}{ }_{-n,-\lambda}\left(-h_{j}^{s}\right)+\left(1-\delta_{s}^{N}\right) B_{Q j}^{f s} \boldsymbol{M}^{\prime}{ }_{-n,-\lambda}\left(-h_{j+2}^{s}\right)\right] \\
& +\left(1-\delta_{f}^{N}\right) \boldsymbol{U}_{n, \lambda}\left(h_{j}^{f}\right)\left[\left(1-\delta_{s}^{1}\right) A_{U j}^{f s} \boldsymbol{N}^{\prime}{ }_{-n t,-\lambda}\left(-h_{j}^{s}\right)+\left(1-\delta_{s}^{N}\right) B_{U j}^{f s} \boldsymbol{N}^{\prime}{ }_{-n t,-\lambda}\left(-h_{j+2}^{s}\right)\right] \\
& \left.+\left(1-\delta_{f}^{N}\right) \boldsymbol{V}_{n, \lambda}\left(h_{j}^{f}\right)\left[\left(1-\delta_{s}^{1}\right) A_{V j}^{f s} \boldsymbol{N}_{-n z,-\lambda}^{\prime}\left(-h_{j}^{s}\right)+\left(1-\delta_{s}^{N}\right) B_{V j}^{f s} \boldsymbol{N}_{-n z,-\lambda}^{\prime}\left(-h_{j+2}^{s}\right)\right]\right\},
\end{aligned}
$$

and

$$
\begin{aligned}
\overline{\boldsymbol{G}}_{3}+ & \overline{\boldsymbol{G}}_{4}=\frac{\mathrm{i}}{2 \pi} \int_{0}^{\infty} \mathrm{d} \lambda \sum_{n=0}^{\infty} \frac{\left(2-\delta_{n 0}\right)}{p_{s} \lambda\left(h_{3 s}-h_{4 s}\right)} \times \sum_{j=3}^{4} \frac{(-1)^{j+1}}{\left(h_{1 s}-h_{j s}\right)\left(h_{2 s}-h_{j s}\right)} \\
& \times\left\{\left(1-\delta_{f}^{N}\right) \boldsymbol{M}_{n, \lambda}\left(h_{j}^{f}\right)\left[\left(1-\delta_{s}^{1}\right) A_{M j}^{f s} \boldsymbol{P}^{\prime}{ }_{-n,-\lambda}\left(-h_{j-2}^{s}\right)+\left(1-\delta_{s}^{N}\right) B_{M j}^{f s} \boldsymbol{P}^{\prime}{ }_{-n,-\lambda}\left(-h_{j}^{s}\right)\right]\right. \\
& +\left(1-\delta_{f}^{N}\right) \boldsymbol{Q}_{n, \lambda}\left(h_{j}^{f}\right)\left[\left(1-\delta_{s}^{1}\right) A_{Q j}^{f s} \boldsymbol{M}^{\prime}{ }_{-n,-\lambda}\left(-h_{j-2}^{s}\right)+\left(1-\delta_{s}^{N}\right) B_{Q j}^{f s} \boldsymbol{M}^{\prime}{ }_{-n,-\lambda}\left(-h_{j}^{s}\right)\right] \\
& +\left(1-\delta_{f}^{N}\right) \boldsymbol{U}_{n, \lambda}\left(h_{j}^{f}\right)\left[\left(1-\delta_{s}^{1}\right) A_{U j}^{f s} \boldsymbol{N}^{\prime}{ }_{-n t,-\lambda}\left(-h_{j-2}^{s}\right)+\left(1-\delta_{s}^{N}\right) B_{U j}^{f s} \boldsymbol{N}^{\prime}{ }_{-n t,-\lambda}\left(-h_{j}^{s}\right)\right] \\
& \left.+\left(1-\delta_{f}^{N}\right) \boldsymbol{V}_{n, \lambda}\left(h_{j}^{f}\right)\left[\left(1-\delta_{s}^{1}\right) A_{V j}^{f s} \boldsymbol{N}^{\prime}{ }_{-n z,-\lambda}\left(-h_{j-2}^{s}\right)+\left(1-\delta_{s}^{N}\right) B_{V j}^{f s} \boldsymbol{N}^{\prime}{ }_{-n z,-\lambda}\left(-h_{j}^{s}\right)\right]\right\} .
\end{aligned}
$$

The combination of the two terms for the above two equations is due to the fact that each term has a static contribution to the dyadic Green's function because of the integration associated with the pole point $\lambda=0$. What should be noted is that the multiple reflection and transmission effects have been included in the formulation of the scattering DGFs. The Sommerfeld radiation condition has been taken into account in the construction of DGFs. The contributions from various wave modes to the DGFs have been considered as well.

\section{Coefficients of the scattering DGFs: boundary condition method}

The boundary conditions that must be satisfied by the dyadic Greens' function at the interface between regions $f$ and $f+1$ where $z=z_{j}=\sum_{l=f}^{N-2} H_{l}$ are shown as follows:

$$
\begin{aligned}
& \widehat{\boldsymbol{z}} \times \overline{\boldsymbol{G}}_{e}^{(f s)}\left(\boldsymbol{r}, \boldsymbol{r}^{\prime}\right)=\widehat{\boldsymbol{z}} \times \overline{\boldsymbol{G}}_{e}^{[(f+1) s]}\left(\boldsymbol{r}, \boldsymbol{r}^{\prime}\right), \\
& \widehat{\boldsymbol{z}} \times\left[\overline{\boldsymbol{\alpha}}_{f} \cdot \nabla \times \overline{\boldsymbol{G}}_{e}^{(f s)}\left(\boldsymbol{r}, \boldsymbol{r}^{\prime}\right)-\omega \xi_{c f} \overline{\boldsymbol{G}}_{e}^{(f s)}\left(\boldsymbol{r}, \boldsymbol{r}^{\prime}\right)\right] \\
& =\widehat{\boldsymbol{z}} \times\left[\overline{\boldsymbol{\alpha}}_{f+1} \cdot \nabla \times \overline{\boldsymbol{G}}_{e}^{[(f+1) s]}\left(\boldsymbol{r}, \boldsymbol{r}^{\prime}\right)-\omega \xi_{c(f+1)} \overline{\boldsymbol{G}}_{e}^{[(f+1) s]}\left(\boldsymbol{r}, \boldsymbol{r}^{\prime}\right)\right] .
\end{aligned}
$$


To simplify the derivation of the general solution of the coefficients, we rewrite the boundary conditions (42) into the following matrix form. Now, it is clear that the equations obtained here for the layered gyrotropic chiral medium are different from those in all previous work.

Besides, it should be pointed out that some relationships between the vectors $\boldsymbol{N}_{n, \lambda}(h), \boldsymbol{M}_{n, \lambda}(h)$ and the matrix $\overline{\boldsymbol{\alpha}}$ have the following form:

$$
\begin{aligned}
& \overline{\boldsymbol{\alpha}} \cdot \boldsymbol{N}_{n, \lambda}(h)=-\frac{\mathrm{i} h}{k_{\lambda}} \alpha_{a} \boldsymbol{M}_{n, \lambda}(h)+\alpha_{t} \boldsymbol{N}_{n t, \lambda}(h)+\alpha_{z} \boldsymbol{N}_{n z, \lambda}(h), \\
& \overline{\boldsymbol{\alpha}} \cdot \boldsymbol{M}_{n, \lambda}(h)=\alpha_{t} \boldsymbol{M}_{n, \lambda}(h)-\frac{\mathrm{i} k_{\lambda}}{h} \alpha_{a} \boldsymbol{N}_{n t, \lambda}(h)+\frac{\mathrm{i} h}{k_{\lambda}} \alpha_{a} \boldsymbol{N}_{n z, \lambda}(h),
\end{aligned}
$$

which will be used in the implementation of (42).

We should also note that some terms such as $\nabla \times Q$ will be generated when applying the boundary condition, hence we give the forms of $\nabla \times Q, \nabla \times U$ and $\nabla \times V$ as follows:

$$
\begin{aligned}
& \nabla \times Q_{n, \lambda}(h)=\frac{h^{2}\left(\tau_{2}-\tau_{3}\right)+k_{\lambda}^{2} \tau_{3}}{k_{\lambda}} M_{n, \lambda}(h), \\
& \nabla \times U_{n, \lambda}(h)=\frac{h^{2}\left(\tau_{5}-\tau_{4}\right)+k_{\lambda}^{2} \tau_{4}}{k_{\lambda}} M_{n, \lambda}(h), \\
& \nabla \times \boldsymbol{V}_{n, \lambda}(h)=\frac{h^{2}\left(\tau_{4}-\tau_{7}\right)+k_{\lambda}^{2} \tau_{7}}{k_{\lambda}} M_{n, \lambda}(h) .
\end{aligned}
$$

\subsection{Recurrence matrix of DGFs' scattering coefficients}

By using the boundary conditions, a set of linear equations of the coefficients which can be represented by a series of compact matrices is given below:

$$
\left[F_{l j^{\prime}(f+1)}\right] \cdot\left\{\left[\Upsilon_{l j^{\prime}(f+1) s}\right]+\delta_{f+1}^{s}\left[U_{(f+1)}\right]\right\}=\left[F_{l j^{\prime} f}\right] \cdot\left\{\left[\Upsilon_{l j^{\prime} f s}\right]+\delta_{f}^{s}\left[D_{f}\right]\right\}
$$

where $j^{\prime}=1,2$ and $l$ denotes $M, Q, U$ and $V$, respectively. These matrices are given by

$$
\begin{aligned}
{\left[F_{M 1 f}\right]=} & {\left[\begin{array}{cc}
\frac{\mathrm{e}^{\mathrm{i} h_{1 f} z_{f}}}{\left(h_{1 s}-h_{2 s}\right)\left(h_{1 s}-h_{4 s}\right)} & \frac{\mathrm{e}^{\mathrm{i} h_{3 f} z_{f}}}{\left(h_{3 s}-h_{4 s}\right)\left(h_{2 s}-h_{3 s}\right)} \\
\frac{\left[h_{1 f} \alpha_{t f}-\left(\omega \xi_{c f}+\mathrm{i} h_{1 f} \alpha_{a f}\right)\right] \mathrm{e}^{\mathrm{i} h_{1 f} z_{f}}}{\left(h_{1 s}-h_{2 s}\right)\left(h_{1 s}-h_{4 s}\right)} & \frac{\left[h_{3 f} \alpha_{t f}-\left(\omega \xi_{c f}+\mathrm{i} h_{3 f} \alpha_{a f}\right)\right] \mathrm{e}^{\mathrm{i} h_{3 f} z_{f}}}{\left(h_{3 s}-h_{4 s}\right)\left(h_{2 s}-h_{3 s}\right)}
\end{array}\right], } \\
{\left[F_{Q 1 f}\right]=} & {\left[\begin{array}{l}
\frac{h_{1 f} \tau_{21 f} \mathrm{e}^{\mathrm{i} h_{1 f} z_{f}}}{k_{\lambda 1 f}\left(h_{1 s}-h_{2 s}\right)\left(h_{1 s}-h_{4 s}\right)} \\
\frac{h_{3 f} \tau_{23 f} \mathrm{e}^{\mathrm{i} h_{3 f} z_{f}}}{k_{\lambda 3 f}\left(h_{3 s}-h_{4 s}\right)\left(h_{2 s}-h_{3 s}\right)} \\
\frac{\left[\left(\tau_{21 f}-\tau_{31 f}\right)\left(\alpha_{t f}-\alpha_{a f}\right) h_{1 f}^{2}+\tau_{31 f}\left(\alpha_{t f}-\alpha_{a f}\right) k_{\lambda 1 f}^{2}-\tau_{21 f} \omega \xi_{c f} h_{1 f}\right] \mathrm{e}^{\mathrm{i} h_{1 f} z_{f}}}{k_{\lambda 1 f}\left(h_{1 s}-h_{2 s}\right)\left(h_{1 s}-h_{4 s}\right)} \\
\\
\frac{\left[\left(\tau_{23 f}-\tau_{33 f}\right)\left(\alpha_{t f}-\alpha_{a f}\right) h_{3 f}^{2}+\tau_{33 f}\left(\alpha_{t f}-\alpha_{a f}\right) k_{\lambda 3 f}^{2}-\tau_{23 f} \omega \xi_{c f} h_{3 f}\right] \mathrm{e}^{\mathrm{i} h_{3 f} z_{f}}}{k_{\lambda 3 f}\left(h_{3 s}-h_{4 s}\right)\left(h_{2 s}-h_{3 s}\right)}
\end{array}\right] }
\end{aligned}
$$




$$
\begin{aligned}
& {\left[F_{U 1 f}\right]=\left[\begin{array}{l}
\frac{h_{1 f} \tau_{51 f} \mathrm{e}^{\mathrm{i} h_{1 f} z_{f}}}{k_{\lambda 1 f}\left(h_{1 s}-h_{2 s}\right)\left(h_{1 s}-h_{4 s}\right)} \\
\frac{h_{3 f} \tau_{53 f} \mathrm{e}^{\mathrm{i} h_{3 f} z_{f}}}{k_{\lambda 3 f}\left(h_{3 s}-h_{4 s}\right)\left(h_{2 s}-h_{3 s}\right)}
\end{array}\right.} \\
& \begin{array}{l}
\left.\frac{\left[\left(\tau_{51 f}-\tau_{41 f}\right)\left(\alpha_{t f}-\alpha_{a f}\right) h_{1 f}^{2}+\tau_{41 f}\left(\alpha_{t f}-\alpha_{a f}\right) k_{\lambda 1 f}^{2}-\tau_{51 f} \omega \xi_{c f} h_{1 f}\right] \mathrm{e}^{\mathrm{i} h_{1 f} z_{f}}}{k_{\lambda 1 f}\left(h_{1 s}-h_{2 s}\right)\left(h_{1 s}-h_{4 s}\right)}\right]^{T} \\
\left.\frac{\left[\left(\tau_{53 f}-\tau_{43 f}\right)\left(\alpha_{t f}-\alpha_{a f}\right) h_{3 f}^{2}+\tau_{43 f}\left(\alpha_{t f}-\alpha_{a f}\right) k_{\lambda 3 f}^{2}-\tau_{53 f} \omega \xi_{c f} h_{3 f}\right] \mathrm{e}^{\mathrm{i} h_{3 f} z_{f}}}{k_{\lambda 3 f}\left(h_{3 s}-h_{4 s}\right)\left(h_{2 s}-h_{3 s}\right)}\right]^{-}
\end{array} \\
& {\left[F_{V 1 f}\right]=\left[\begin{array}{c}
\frac{h_{1 f} \tau_{41 f} \mathrm{e}^{\mathrm{i} h_{1 f} z_{f}}}{k_{\lambda 1 f}\left(h_{1 s}-h_{2 s}\right)\left(h_{1 s}-h_{4 s}\right)} \\
\frac{h_{3 f} \tau_{43 f} \mathrm{e}^{\mathrm{i} h_{3 f} z_{f}}}{k_{\lambda 3 f}\left(h_{3 s}-h_{4 s}\right)\left(h_{2 s}-h_{3 s}\right)}
\end{array}\right.} \\
& \begin{array}{l}
\left.\frac{\left[\left(\tau_{41 f}-\tau_{71 f}\right)\left(\alpha_{t f}-\alpha_{a f}\right) h_{1 f}^{2}+\tau_{71 f}\left(\alpha_{t f}-\alpha_{a f}\right) k_{\lambda 1 f}^{2}-\tau_{41 f} \omega \xi_{c f} h_{1 f}\right] \mathrm{e}^{\mathrm{i} h_{1 f} z_{f}}}{k_{\lambda 1 f}\left(h_{1 s}-h_{2 s}\right)\left(h_{1 s}-h_{4 s}\right)}\right]^{T} \\
\left.\frac{\left[\left(\tau_{43 f}-\tau_{73 f}\right)\left(\alpha_{t f}-\alpha_{a f}\right) h_{3 f}^{2}+\tau_{73 f}\left(\alpha_{t f}-\alpha_{a f}\right) k_{\lambda 3 f}^{2}-\tau_{43 f} \omega \xi_{c f} h_{3 f}\right] \mathrm{e}^{\mathrm{i} h_{3 f} z_{f}}}{k_{\lambda 3 f}\left(h_{3 s}-h_{4 s}\right)\left(h_{2 s}-h_{3 s}\right)}\right]^{2},
\end{array}
\end{aligned}
$$

where the superscript $T$ denotes the transpose of the matrices.

The matrices $\left[F_{l j^{\prime} f}\right]$ remain the same form for $j^{\prime}=2$ except the subscript 1 is changed to 2 and the subscript 3 is changed to 4 . Furthermore, the denominator which contains the term $\left(h_{1 s}-h_{4 s}\right)$ is changed to $\left(h_{2 s}-h_{3 s}\right)$ and vice versa. The terms $\tau_{2 j f}, \tau_{3 j f}, \tau_{4 j f}, \tau_{5 j f}$ and $\tau_{7 j f}$ are the weighting factors associated with the scattering coefficients $A_{l j}^{f s}$ and $B_{l j}^{f s}$. They have the same forms as those in (29) and (33) only with the change that each term relating to wave numbers (e.g., $h$ ) will have a subscript of $j f$ (e.g., $\left.h_{j f}\right)$ and each term relating to material parameters (e.g., $\xi_{c}$ ) will have a subscript of $f$ (e.g., $\xi_{c f}$ ) where $j=1,2$ and $f$ represents the $f$ th layer.

The following matrices are also used in the formulation:

$$
\begin{aligned}
& {\left[\Upsilon_{l j^{\prime} f s}\right]=\left[\begin{array}{cc}
A_{l j^{\prime}}^{f s} & B_{l j^{\prime}}^{f s} \\
A_{l, j^{\prime}+2}^{f s} & B_{l, j^{\prime}+2}^{f s}
\end{array}\right],} \\
& {\left[U_{f}\right]=\left[\begin{array}{ll}
1 & 0 \\
0 & 0
\end{array}\right],} \\
& {\left[D_{f}\right]=\left[\begin{array}{ll}
0 & 0 \\
0 & 1
\end{array}\right] .}
\end{aligned}
$$

Defining the following transmission $T$-matrix:

$$
\left[T_{l j^{\prime} f}\right]=\left[F_{l j^{\prime}(f+1) f}\right]^{-1} \cdot\left[F_{l j^{\prime} f f}\right],
$$

where $\left[F_{l j^{\prime}(f+1) f}\right]^{-1}$ is the inverse matrix of $\left[F_{l j^{\prime}(f+1) f}\right]$, we can thus rewrite the linear equation into the following form:

$$
\left[\Upsilon_{l j^{\prime}(f+1) s}\right]=\left[T_{l j^{\prime} f}\right] \cdot\left\{\left[\Upsilon_{l j^{\prime} f s}\right]+\delta_{f}^{s}\left[D_{f}\right]\right\}-\delta_{f+1}^{s}\left[U_{(f+1)}\right] .
$$


To shorten the expression, we also introduce

$$
\begin{aligned}
{\left[T_{l j^{\prime}}^{K}\right]_{2 \times 2} } & =\left[T_{l j^{\prime}, N-1}\right]\left[T_{l j^{\prime}, N-2}\right] \cdots\left[T_{l j^{\prime}, K+1}\right]\left[T_{l j^{\prime}, K}\right] \\
& =\left[\begin{array}{ll}
T_{l j^{\prime}, 11}^{K} & T_{l j^{\prime}, 12}^{K} \\
T_{l j^{\prime}, 21}^{K} & T_{l j^{\prime}, 22}^{K}
\end{array}\right] .
\end{aligned}
$$

Note that the coefficients matrices of the first and the last layers have the following relations:

$$
\left[\Upsilon_{l j^{\prime} 1 s}\right]=\left[\begin{array}{cc}
A_{l j^{\prime}}^{1 s} & B_{l j^{\prime}}^{1 s} \\
0 & 0
\end{array}\right], \quad\left[\Upsilon_{l j^{\prime} N s}\right]=\left[\begin{array}{cc}
0 & 0 \\
A_{l, j^{\prime}+2}^{N s} & B_{l, j^{\prime}+2}^{N s}
\end{array}\right] .
$$

\subsection{Specific applications: three cases}

To gain insight into the specific mathematical expressions of the physical quantities such as the transmission and reflections coefficient matrices, the following three cases are specifically considered where the source point is located in the first, the intermediate and the last layers.

4.2.1. Source in the first layer. When the current source is located in the first layer (i.e., $s=1)$, the terms containing $\left(1-\delta_{s}^{1}\right)$ in (40) and (41) vanish. The coefficient matrices in (53) and (59) will be further reduced to

$$
\begin{aligned}
& {\left[\Upsilon_{l j^{\prime}, 11}\right]=\left[\begin{array}{cc}
0 & B_{l j^{\prime}}^{11} \\
0 & 0
\end{array}\right],} \\
& {\left[\Upsilon_{l j^{\prime}, m 1}\right]=\left[\begin{array}{cc}
0 & B_{l j^{\prime}}^{m 1} \\
0 & B_{l, j^{\prime}+2}^{m 1}
\end{array}\right],} \\
& {\left[\Upsilon_{l j^{\prime}, N 1}\right]=\left[\begin{array}{cc}
0 & 0 \\
0 & B_{l, j^{\prime}+2}^{N 1}
\end{array}\right],}
\end{aligned}
$$

where $m=2,3, \ldots, N-1$. It can be seen that only four coefficients for the first layer and the last layer, but 8 coefficients for each of the remaining layers, need to be solved. By following (57), the recurrence relation in the $f$ th layer becomes

$$
\left[\Upsilon_{l j^{\prime}, f 1}\right]=\left[T_{l j^{\prime}, f-1}\right] \cdots\left[T_{l j^{\prime}, 1}\right]\left\{\left[\Upsilon_{l j^{\prime}, 11}\right]+\left[D_{1}\right]\right\} .
$$

With $f=N$ in (61), a matrix equation satisfied by the coefficient matrices in (60) can be obtained. The coefficient for the first layer where the source is (i.e. $s=1)$ is given by

$$
B_{l j^{\prime}}^{11}=-\frac{T_{l j^{\prime}, 12}^{(1)}}{T_{l j^{\prime}, 11}^{(1)}} .
$$

The coefficient for the last layer can be derived in terms of the coefficients for the first layer given by

$$
B_{l, j^{\prime}+2}^{N 1}=T_{l j^{\prime}, 21}^{(1)} B_{l j^{\prime}}^{11}+T_{l j^{\prime}, 22}^{(1)}
$$

The coefficients for the intermediate layers can be then obtained by substituting the coefficients for the first layer in (62) to (61). Thus, all the coefficients can be obtained by these procedures. 
4.2.2. Source in the intermediate layers. When the current source is located in an intermediate layer, (i.e. $s \neq 1, N)$, only the terms containing $\left(1-\delta_{f}^{1}\right)$ for the first layer and $\left(1-\delta_{f}^{N}\right)$ for the last layer vanish in (40) and (41). The coefficient matrices in (53) and (59) will be further simplified:

$$
\begin{aligned}
& {\left[\Upsilon_{l j^{\prime}, 1 s}\right]=\left[\begin{array}{cc}
A_{l j^{\prime}}^{1 s} & B_{l j^{\prime}}^{1 s} \\
0 & 0
\end{array}\right],} \\
& {\left[\Upsilon_{l j^{\prime}, m s}\right]=\left[\begin{array}{cc}
A_{l j^{\prime}}^{m s} & B_{l j^{\prime}}^{m s} \\
A_{l, j^{\prime}+2}^{m s} & B_{l, j^{\prime}+2}^{m s}
\end{array}\right],} \\
& {\left[\Upsilon_{l j^{\prime}, N s}\right]=\left[\begin{array}{cc}
0 & 0 \\
A_{l, j^{\prime}+2}^{N s} & B_{l, j^{\prime}+2}^{N s}
\end{array}\right] .}
\end{aligned}
$$

From (57), the recurrence equation becomes

$$
\begin{gathered}
{\left[\Upsilon_{l j^{\prime}, f s}\right]=\left[T_{l j^{\prime}, f-1}\right] \cdots\left[T_{l j^{\prime}, s}\right] \cdot\left\{\left[T_{l j^{\prime}, s-1}\right] \cdots\left[T_{l j^{\prime}, 1}\right]\left[\Upsilon_{l j^{\prime}, 1 s}\right]\right.} \\
\left.+u(f-s-1)\left[D_{s}\right]-u(f-s)\left[U_{s}\right]\right\},
\end{gathered}
$$

where $u\left(x-x_{0}\right)$ is the unit step function. For $f=N$, the coefficients for the first layer are given by

$$
\begin{aligned}
& A_{l j^{\prime}}^{1 s}=\frac{T_{l j^{\prime}, 11}^{(s)}}{T_{l j^{\prime}, 11}^{(1)}}, \\
& B_{l j^{\prime}}^{1 s}=-\frac{T_{l j^{\prime}, 12}^{(s)}}{T_{l j^{\prime}, 11}^{(1)}} .
\end{aligned}
$$

For the last layer,

$$
\begin{aligned}
& A_{l, j^{\prime}+2}^{N s}=T_{l j^{\prime}, 21}^{(1)} A_{l j^{\prime}}^{1 s}-T_{l j^{\prime}, 21}^{(s)}, \\
& B_{l, j^{\prime}+2}^{N s}=T_{l j^{\prime}, 21}^{(1)} B_{l j^{\prime}}^{1 s}+T_{l j^{\prime}, 22}^{(s)} .
\end{aligned}
$$

Substituting (66) into (65), the rest of the coefficients can be obtained for the dyadic Green's functions.

4.2.3. Source in the last layer. When the current source is located in the first layer (i.e., $s=N)$, the coefficients are

$$
\begin{aligned}
& {\left[\Upsilon_{l j^{\prime}, 1 N}\right]=\left[\begin{array}{cc}
A_{l j^{\prime}}^{1 N} & 0 \\
0 & 0
\end{array}\right],} \\
& {\left[\Upsilon_{l j^{\prime}, m N}\right]=\left[\begin{array}{cc}
A_{l j^{\prime}}^{m N} & 0 \\
A_{l, j^{\prime}+2}^{m N} & 0
\end{array}\right],} \\
& {\left[\Upsilon_{l j^{\prime}, N N}\right]=\left[\begin{array}{cc}
0 & 0 \\
A_{l, j^{\prime}+2}^{N N} & 0
\end{array}\right] .}
\end{aligned}
$$

From the recurrence equation (57), similarly we have

$$
\left[\Upsilon_{l j^{\prime}, f N}\right]=\left[T_{l j^{\prime}, f-1}\right] \cdots\left[T_{l j^{\prime}, 1}\right]\left[\Upsilon_{l j^{\prime}, 1 N}\right]-u(f-N)\left[U_{N}\right] .
$$

By letting $f=N$, the coefficient for the first region is

$$
A_{l j^{\prime}}^{1 N}=\frac{1}{T_{l j^{\prime}, 11}^{(1)}} \text {. }
$$


And for the last layer, it is found that

$$
A_{l, j^{\prime}+2}^{N N}=T_{l j^{\prime}, 21}^{(1)} A_{l j^{\prime}}^{1 N} .
$$

Similarly, the rest of the coefficients can be obtained by inserting (73) into (71).

So far, for gyrotropic chiral media in planarly multilayered structure, We have obtained a complete set of the DGFs in terms of the cylindrical vector wavefunctions and their scattering coefficients in terms of compact matrices. Reduction can be made for formulating the dyadic Green's functions in a less complex medium of specific planar geometries, e.g., an anisotropic medium where $\xi_{c}=0$, a chiral medium where $g=w=0, \epsilon=\epsilon_{z}$ and $\mu=\mu_{z}$, a gyroelectric medium where $w=0$ and $\mu=\mu_{z}$, a chiroferrite medium where $p=0$ and $\epsilon=\epsilon_{z}$, an isotropic medium where $\xi_{c}=g=w=0, \epsilon_{z}=\epsilon$ and $\mu=\mu_{z}$. When the source distribution is given, the electric field can be computed by (4), in which the local parameters of the source, the stratified layers and the materials in each layer have been taken into account systematically.

\section{Conclusion}

Gyrotropic chiral media, which can achieve negative refraction far off the resonances of the permittivity and permeability, provide us a promising alternative approach to realize negativerefractive media for optical applications. In this paper, systematic response of gyrotropic chiral media has been studied in terms of the dyadic Green's functions. A complete eigenfunctional expansion of the DGFs for an unbounded gyrotropic chiral medium and stratified multilayered gyrotropic chiral media is constructed. The unbounded DGFs in the gyrotropic chiral medium are obtained using the $\mathrm{Ohm}-$ Raleigh method, and the scattered dyadics are then proposed using the principles of scattering superposition for a multilayered structure as well as the multiple transmission and reflection associated with each interface. The scattering coefficients of the DGFs are presented in compact matrices by applying the boundary conditions at each interface. The current form of DGFs for gyrotropic chiral media includes the irrotational dyadic, which has been extracted in this paper. It is worth pointing out that (1) the general DGFs for the planarly multilayered gyrotropic chiral media can be reduced to those DGFs for less complex media as expected and mentioned before, such as chiral media, anisotropic media, gyroelectric media, chiroferrite media and isotropic media; (2) various wave modes containing different wave numbers $h_{1}, h_{2}, h_{3}$ and $h_{4}$ are observed during the formulation of the DGFs; and (3) thus, the DGFs can be decomposed using the modes mentioned above. Application of the present work can be made to problems of propagation and scattering and dipole antenna radiation in the planarly multilayered gyrotropic chiral media.

\section{Acknowledgments}

The authors are grateful to financial support from the SUMMA Foundation, US 2006. Also, the partial support from France-Singapore Merlion Project is acknowledged.

\section{References}

[1] Veselago V G 1968 Sov. Phys.-Usp. 10509

[2] Pendry J B 2000 Phys. Rev. Lett. 853966

[3] Shelby R A, Smith D R and Schultz S 2001 Science 29277

[4] Ishimaru A, Lee S W, Kuga Y and Jandhyala V 2003 IEEE Trans. Antennas Propag. 512550

[5] Chen X, Wu B I, Kong J A and Grzegorczyk T M 2005 Phys. Rev. E 71046610

[6] Eleftheriades G V, Iyer A K and Kremer P C 2002 IEEE Trans. Microwave Theory Tech. 502702

[7] Lai A, Itoh T and Caloz C 2004 IEEE Microwave Magazine 534 
[8] Qiu C W, Yao H Y, Li L W, Zouhdi S and Yeo T S 2007 Phys. Rev. B 75755120

[9] Qiu C W, Yao H Y, L. W. Li, Yeo T S and Zouhdi S 2007 Phys. Rev. B at press

[10] Lakhtakia A and Mackay T G 2004 J. Phys. A: Math. Gen. 37505

[11] Qiu C W, Yao H Y, Li L W, Zouhdi S and Leong M S 2006 Microw. Opt. Tech. Lett. 482534

[12] He S, Jin Y, Ruan Z and Kuang J 2005 New J. Phys. 7210

[13] Marqés R, Baena J, Beruete M, Falcone F, Lopetegi T, Sorolla M, Martín F and Garcia J 2005 J. Opt. A: Pure Appl. Opt. 738

[14] Hsu Y J, Huang Y C, Lih J S and Chern J L 2004 J. Appl. Phys. 961979

[15] Zhang F L, Zhao Q, Liu Y H, Luo C R and Zhao X P 2004 Chin. Phys. Lett. 211330

[16] Tretyakov S, Nefedov I, Sihvola A, Maslovski S and Simovski C 2003 J. Electromagn. Waves Appl. 17695

[17] Shen J Q and He S 2006 J. Phys. A: Math. Gen. 39457

[18] Qiu C W and Zouhdi S 2007 Phys. Rev. B at press

[19] Tai C T 1994 Dyadic Green's Functions in Electromagnetic Theory 2nd edn (Piscataway, NJ: IEEE)

[20] Kong J A 1990 Electromagnetic Wave Theory 3rd edn (New York: Wiley)

[21] Kong J A 1972 Geophys. 37985

[22] Stoyer C H 1977 IEEE Trans. Antennas Propag. 25547

[23] Ali S M and Mahmoud S F 1979 IEEE Trans. Antennas Propag. 27671

[24] Li L W, Koh J H, Yeo T S, Leong M S and Kooi P S 1999 IEEE Trans. Geosci. Remote Sensing 371967

[25] Koh J H, Li L W, Kooi P S, Yeo T S and Leong M S 1999 Radio Sci. 34681

[26] Cavalcante G P S, Rogers D A and Giardola A J 1982 Radio Sci. 7503

[27] Cavalcante G P S and Giardola A J 1983 IEEE Trans. Antennas Propag. 31141

[28] Li L W, Yeo T S, Kooi P S and Leong M S 2000 IEEE Trans. Antennas Propag. 481220

[29] Collin R E 1991 Field Theory of Guided Waves 2nd edn (Piscataway, NJ: IEEE)

[30] Lee J K and Kong J A 1983 Electromagnetics 3111

[31] Lee J K and Kong J A 1985 IEEE Trans. Geosci. Remote Sensing 23910

[32] Krowne C M 1989 IEEE Trans. Antennas Propag. 371207

[33] Ren W 1993 Phys. Rev. E 47664

[34] Weiglhofer W S and Lindell I V 1994 AEU 48116

[35] Chew W C 1990 Waves and Fields in Inhomogeneous Media (New York: Van Nostrand)

[36] Li L W, Lim N H, Leong M S, Yeo T S and Kong J A 2000 Microwave Conference, 2000 Asia-Pacific, pp 303-6

[37] Li K and Pan W Y 2002 Chin. Phys. 111245

[38] Cheng D 1997 Phys. Rev. E 551950

[39] Li L W, Leong M S, Yeo T S and Kooi P S 1999 Phys. Rev. E 593767

[40] Weiglhofer W S 1990 IEEE Proc. H 1375

[41] Engheta N, Jaggard D L and Kowarz M W 1992 IEEE Trans. Antennas Propag. 40367

[42] Li L W, Koh J H, Yeo T S, Leong M S and Kooi P S 2004 IEEE Trans. Antennas Propag. 52466

[43] Cheng D, Ren W and Jin Y Q 1997 J. Phys. A: Math. Gen. 30573

[44] Cheng D and Antar Y M M 1997 Phys. Rev. E 567273

[45] Kruchinin S P 1995 Mod. Phys. Lett. B 9379 